\title{
Crystallography and dynamics in superspace
}

\author{
Céline Mariette, Philippe Rabiller, Laurent Guérin, and Bertrand Toudic \\ Institute of Physics Rennes, U.M.R. CNRS 6251, University of Rennes 1, 91944 Rennes, France
}

\begin{abstract}
The discovery in materials of long range order without lattice periodicity has generated a very broad field of research with the introduction of totally new concepts. The common feature of this new state of matter is the translational symmetry recovered by adding supplementary dimensions to the physical space, defining a crystallographic superspace. Along these supplementary directions, specific low excitations, so-called phasons, are predicted and observed. These quasi-periodic crystals may present phase transitions as function of external parameters, which have then to be described as symmetry breakings within these crystallographic superspaces. Three different families of quasi-periodic crystals are usually considered: incommensurate modulated crystals, aperiodic composites and quasicrystals. Their crystallography and dynamics are discussed.
\end{abstract}

\section{Introduction}

In the last part of the $\mathrm{XX}^{\text {th }}$ century, the definition of the crystal changed. The translation symmetry is no more a condition. Only the existence of long range order is required. Of course, the lost of the translation symmetry has severe consequences on the determination of the properties of these so-called aperiodic crystals: where are the atoms?, what about the phase transitions in these crystals?, how to describe the collective vibrations in a crystal with no unit cell and so no Brillouin zone? The goal of this paper is to present the conceptual advances done in these fields up to now. The case of the three families of aperiodic crystals will be treated: incommensurate modulated crystals, aperiodic composites and quasicrystals.

\section{Periodic crystals}

The periodic crystal is defined by the identical repetition in the three dimensions of space of an elementary unit-cell, characterized by three independent basic vectors $a, b$ and $c$. The existence of the translation symmetry, defined by the translational vector $\mathbf{T}=\mathrm{n}_{1} \mathbf{a}+\mathrm{n}_{2} \mathbf{b}+\mathrm{n}_{3} \mathbf{c}$, is at the origin of the huge simplification met in solid state physics. Indeed, this brings a problem of 1022 atoms for a crystal of $1 \mathrm{~cm}^{3}$ to a problem of the few $\mathrm{N}$ atoms in the unit cell, $\mathrm{N}$ varying from 1 (in Polonium) to some 104 in proteins crystals. The translation symmetry allows the introduction of the following concepts:

- the reciprocal space $\mathbf{G}$, such as $\mathbf{G} . \mathbf{T}=2 \pi, \mathbf{G}=\mathbf{G}_{h k l}=$ ha* $\mathbf{a}^{*} \mathbf{k b}^{*}+\mathbf{c}^{*}$ where $\mathbf{a}^{*}, \mathbf{b}^{*}$ and $\mathbf{c}^{*}$ are the vectors of the dual space of the real space, called the reciprocal space.

- the structure factor associated to the nodes $\mathbf{G}_{\mathrm{hkl}}$ of this reciprocal space, is simply obtained by a sum limited to the $\mathrm{N}$ atoms in the positions $\mathbf{r}_{j}$ in the unit cell: $F\left(\boldsymbol{G}_{\boldsymbol{h k l}}\right)=$ $\sum_{j=1}^{N} f_{j} e^{i \boldsymbol{G} \cdot \boldsymbol{r}_{j}}$, where $f_{j}$ is the scattering potential of the atom $\mathrm{j}$. 
- the Brillouin zone which is the primitive cell in the reciprocal space. Within this Brillouin zone the collective dynamics of the atoms of the crystal is described in term of $3 \mathrm{~N}$ phonon branches. Three branches are acoustic branches corresponding in the Brillouin zone center to the 3 Goldstone modes expected in a space of dimension $\mathrm{D}=3$, and $(3 \mathrm{~N}-3)$ branches are optic branches. Taking advantage again from the translation symmetry, the description of the electronic bands, the optical and magnetic properties... may also be done within this Brillouin zone.

An important question in solid state physics concerns the phase transformations that crystals may present under external fields (temperature, pressure, electric or magnetic field, light, ...). Describing these phase transitions in matter is determining the cooperative change of a macroscopic system made of a huge number of particles. This problem is of an extreme complexity even in the periodic crystals because the cooperative fluctuations are much longer than the size of the elementary cell. These fluctuations actually diverge in the space and in time at the transition, creating by this way a new macroscopic order. The description of these pretransitional phenomena leading to the symmetry breaking is modelled taking into account:

- the dimension of the physical space.

- the dimensionality of the order parameter $(n=1$ in the Ising-model, $n=2$ in the $X-Y$ planer model, $\mathrm{n}=3$ in the Heisenberg model).

- the range of the involved interactions.

These criteria define universality classes. Actually, very few of these models are soluble and, in the 70's, another approach, called group renormalization theory, has allowed the description of the set of variables associated to a phase transition. This method gives access to the values of the critical exponents associated to these different universality classes (Wilson, Fisher, Kadanoff, Nobel Prize 1972). Here, we will not consider such critical approaches, limiting the discussion to the mean field phenomenological approach proposed by Landau and corrected later on by Ginsburg and Levanyuk in order to take into account the pretransitionnal fluctuations [1-5]. The Landau theory does not try to describe the real neighbour-neighbour interaction, but instead introduce a mean effective field applied to a single given particle. In this theory, an order parameter $\eta$ is defined as the measure of the loss of the symmetry element associated to a symmetry breaking at the transition temperature $\mathrm{T}_{c}$. Spatial fluctuations of the order parameters correspond to the appearance of a short range order on correlation lengths usually anisotropic $\xi_{\mathrm{a}}, \xi_{\mathrm{b}}, \xi_{\mathrm{c}}$. To this local order is associated a temporal fluctuation, lifetime $\tau$ of these local fluctuations $\eta(\boldsymbol{r}, t)$ In the high symmetry phase, $\langle\eta(\boldsymbol{r}, t)\rangle_{t=\infty}=\bar{\eta}=0$ but the fluctuations $\left\langle\eta^{2}\right\rangle$ are non-zero very close to the transition temperature: $\langle\eta(\boldsymbol{r}, t)\rangle_{\xi} \neq 0$ and $\langle\eta(\boldsymbol{r}, t)\rangle_{\tau} \neq 0$. Critical diffuse scattering is measured in the reciprocal space around the critical wave vectors which signs the symmetry breaking. It is described by the Orstein-Zernicke expression, Fourier transform of the pair correlation function:

$$
S(\boldsymbol{q}, T) \sim \frac{k_{B} T}{1+\xi_{a}^{2} q_{x}^{2}+\xi_{b}^{2} q_{y}^{2}+\xi_{c}^{2} q_{z}^{2}}
$$

The analysis of experimental profiles of the diffuse scattering $S(\boldsymbol{q}, T)$ allows the extraction of the experimental correlation lengths. The lifetime of these correlations is measured at these same locations in the reciprocal space, usually by inelastic neutron scattering techniques. 


\section{Aperiodic crystals}

\subsection{Structural aspects}

If we limit to structural aspects, there exists three families of aperiodic crystals: the incommensurate modulated phases, the aperiodic composites and the quasicrystals. The loss of the periodicity of a crystalline system has the major consequence that the theoretical models defining the physical properties cannot be based on the Bloch theorem. To recover the lost periodicity, the concept of superpace crystallography has been introduced by de Wolf, Janner and Janssen [6-9]. In a general way, the superspace has a dimension, or rank, $\mathrm{n}=\mathrm{D}+\mathrm{d}$, where $\mathrm{D}$ is the dimension of the physical space (lower or equal to 3 ) and $\mathrm{d}$ the dimension of the internal space of the superspace. The cost of such creation is that the atom is no more defined by the three coordinates $\mathbf{r}_{\mathrm{j}}\left(\mathrm{x}_{\mathrm{j}}, \mathrm{y}_{\mathrm{j}}, \mathrm{z}_{\mathrm{j}}\right)$ of the physical space but becomes a function in a space of dimension larger than the physical one. In a space of dimension 4 , this function is a curve whereas it is a plane and a volume in spaces of rank respectively 5 and 6 $[10,11]$. These different concepts are presented in several books $[10,12,13]$.

\subsubsection{The incommensurate modulated phases}

The modulated incommensurate compounds can be seen as a perturbation of a periodic tridimensional lattice by a modulation whose period is in an incommensurate ratio with the periodicity of the mean lattice. In a general way, the incommensurate phase appears when there is a frustration between antagonistic forces. The first case observed experimentally concerned a magnetic modulation [14]. They correspond to crystals in which a spin wave exists and presents a periodicity which is incommensurate with the period of the lattice. Since that time, a very large number of atomic modulations have been observed [15] and it appears that in fact these phases are rather current in nature. An important research axis in the incommensurate modulated crystals concerns compounds of low dimensionality presenting Peierls instability. In that case, the charge density waves result from the competition between the elastic energy and the electronic energy related to the partial filling of the Fermi band [16]. To illustrate an incommensurate modulated material, let us consider a chain of atoms of period a, modulated in position by a sinusoidal function $A \sin (q x+\phi)$ where $q=\frac{2 \pi}{\lambda}$ and

$\frac{\lambda}{a}$ is irrational. $\phi$ is the phase of the modulation. By introducing a perpendicular direction to the physical space, we can create along the new direction a function which is the one of the incommensurate modulation. The atom is no more a point but is defined by this atomic function. The position of the atoms in the physical space is obtained by a three-dimensional cut of a superspace of dimension $\mathrm{n}$ (in the Fig. 1, one-dimensional cut of superspace of rank 2 ). The perpendicular space contains the information on the phase of the modulation and any fluctuation along this direction is called phase mode or "phason". In these aperiodic materials, the term phonon is reserved to vibration modes contained in the physical space. In the extreme simplest case, the atomic function is a sinusoidal function. In real crystals, the function is more complex (described then by the presence of harmonics) and can even become discontinuous when position of the atoms are forbidden for instance for steric reasons. Often, one has considered the discontinuous characters of the atomic functions as specific to quasicrystals, which have indeed an image made of limited segments in the crystallographic superspace. The possible existence of an analycity breaking in incommensurately modulated crystals and in aperiodic composites proves that this property is not limited to quasicrystal $[17,18]$. The reciprocal space, dual space of the superspace of dimension $\mathrm{n}$, is also of rang $\mathrm{n}$ and is defined by the generalization of the expression $\boldsymbol{G} . \boldsymbol{T}=2 \pi \boldsymbol{a}_{\boldsymbol{i}} \cdot \boldsymbol{a}_{j}^{*}=2 \pi \delta_{i j}$ with $i, j=1, n$. 


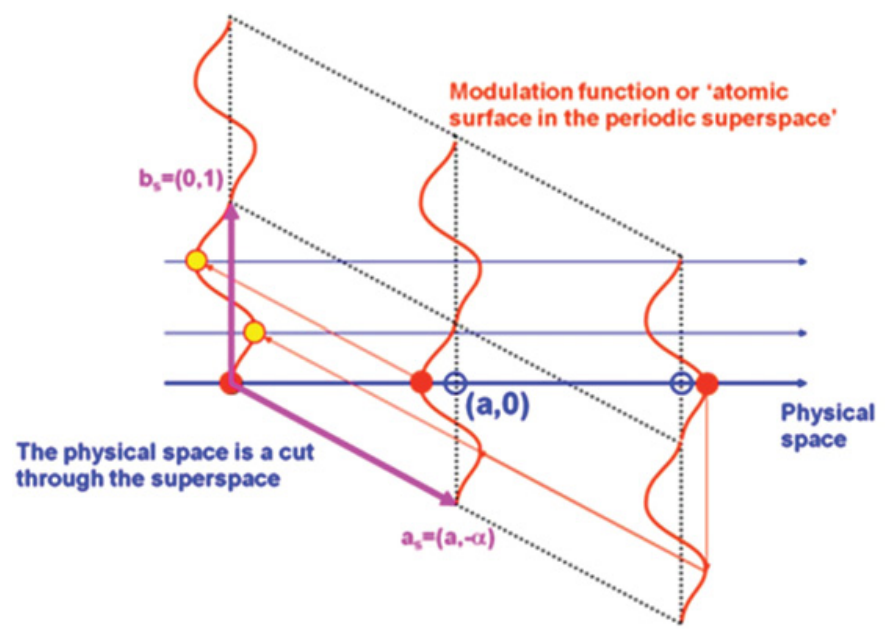

Figure 1. Left: schematic representation of a superspace of dimension $1+1$ in the real space, the orange curve represent the atomic function along the internal dimension of the superspace. The actual positions of the atoms are given by a horizontal cut; right: in the reciprocal space, the actual Bragg positions are obtained by a projection of the reciprocal nodes on the physical reciprocal space.

In the case of incommensurately modulated crystals, there exists a mean structure which is periodic in three dimensions. This allows the definition of a mean Brillouin zone. In the case of an incommensurate modulation characterized by a single vector of modulation, the dimension of the superspace group is four, characterized by the four reciprocal vectors $\mathbf{a}^{*}$, $\mathbf{b}^{*}, \mathbf{c}^{*}$ and $\mathbf{q}_{\mathrm{s}}$. The vectors of the reciprocal space are written:

$$
\boldsymbol{G}_{h k l m}=h \boldsymbol{a}^{*}+k \boldsymbol{b}^{*}+l \boldsymbol{c}^{*}+m \boldsymbol{q}_{s}
$$

In the reciprocal space, one defines two types of Bragg peaks:

- the Bragg peaks (hk10) also called main reflections, associated to the mean structure.

- the satellites (hklm) with $\mathrm{m} \neq 0$ associated to the modulation.

The incommensurate modulated phases results usually from a continuous phase transition. As a consequence, the amplitude A of the modulation is weak at least close to the transition temperature. The calculation of the intensity of the satellite peaks (hklm) with $\mathrm{m} \neq 0$ can be done in simple cases. Let us consider a sinusoidal modulation as described above, the position the $\mathrm{j}^{\text {th }}$ atom in the unit cell is:

$$
\boldsymbol{U}_{j}^{l}=\boldsymbol{R}_{l}+\boldsymbol{r}_{j}+\boldsymbol{A} \sin \left(\boldsymbol{q} \cdot \boldsymbol{R}_{l}+\varphi_{j}\right)
$$

For such a crystal the structure factor is:

$$
F(\boldsymbol{Q})=\sum_{j, l} f_{j} e^{i \boldsymbol{Q} \cdot \boldsymbol{U}_{j}^{l}}=\sum_{j, l} f_{j} e^{i \boldsymbol{Q} \cdot\left(\boldsymbol{R}_{l}+\boldsymbol{r}_{j}\right)} e^{i \boldsymbol{Q} \cdot \boldsymbol{A}\left(\sin \left(\boldsymbol{q} \cdot \mathbf{R}_{l}+\phi_{j}\right)\right.}
$$

According to Auger-Jacobi formula:

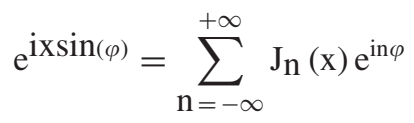


Table 1. Notation of the superspace groups.

\begin{tabular}{|c|c|}
\hline $\begin{array}{c}\text { Symbol of the space group } \\
\text { of the mean structure }\end{array}$ & $\begin{array}{c}\text { Symbol of the modulation vector, } \\
\text { expressed between parenthesis }\end{array}$ \\
\hline Example of $\mathrm{Na} 2 \mathrm{CO} 3$ in dimension $(3+1): \mathrm{C} 2 / \mathrm{m}(\alpha 0 \gamma)$. \\
\hline
\end{tabular}

it comes:

$$
F(\boldsymbol{Q})=\sum_{m=-\infty}^{+\infty}\left(\sum_{j=1}^{N} f_{j} J_{m}(\boldsymbol{Q} \cdot \boldsymbol{A}) e^{i \boldsymbol{Q} \cdot r_{j}} e^{i m \phi_{j}} \sum_{G} \delta(\boldsymbol{Q}+m \boldsymbol{q}-\boldsymbol{G})\right)
$$

The diffracted intensity is then:

$$
I(\boldsymbol{G} \pm m \boldsymbol{q})=\left|\sum_{j} f_{j} J_{m}[(\boldsymbol{G} \pm m \boldsymbol{q}) \cdot \boldsymbol{A}] e^{i(\boldsymbol{G} \pm m \boldsymbol{q}) \cdot \boldsymbol{r}_{j}} e^{i m \phi_{j}}\right|^{2}
$$

For small amplitude A of the modulation, $\mathrm{J}_{\mathrm{m}}(\mathrm{x}) \sim \mathrm{x}_{\mathrm{m}}$ and so $(\boldsymbol{G}+m \boldsymbol{q}) \propto[(\boldsymbol{G}+m \boldsymbol{q}) \cdot A]^{2 m}$ where the term $[(\boldsymbol{G}+m \boldsymbol{q}) . A]$ is much lower than 1. So, in theory, there is an infinite number of Bragg peaks distributed uniformly in the reciprocal space. However, since the intensity of the satellite Bragg peaks decrease very fast with their order m, only the satellites with a very low order are observable. The criterion is even more drastic in the case of an occupation modulation, where the intensity of the satellites is non zero only for the order \pm 1 [12]. In the general case, however, the modulation is not purely sinusoidal and there are no strict rules concerning the intensity of the different satellites peaks. The diffraction image being constituted of a discrete ensemble of reflections, there exist a finite number of symmetry elements leaving it invariant. The whole set of symmetry operations form the group of superspace of rank $\mathrm{D}+\mathrm{d}$. This superspace group is determined by the observation of the reflections conditions discussed here on the $\mathrm{D}+\mathrm{d}$ indices. So a centering $\mathrm{I}$ in a superspace of dimension 4 characterized by the above expression will imply the condition of presence $\mathrm{h}+\mathrm{k}+\mathrm{l}+\mathrm{m}$ even [10]. To correctly define the symmetry of an aperiodic crystal, it is essential to identify what are the basic vectors of the system which will define the name of the the superspace group. They will be constituted of the three base vectors $\mathbf{a}^{*}, \mathbf{b}^{*}$ and $\mathbf{c}^{*}$ characterizing the physical reciprocal space and of " $\mathrm{d}$ " vectors (representing the dimension of the internal space of the superspace) expressed as a function of these three basic vectors. The notation of the superspace groups is derived from the one of the usual space groups writing close to it the three components of the d modulation vectors.

For instance, for sodium carbonate, the first compound studied by de Wolff et al., the mean structure is monoclinic $\mathrm{C} 2 / \mathrm{m}$. The modulation is characterized by the vector $\boldsymbol{q}_{\mathrm{s}}=$ $\alpha \boldsymbol{a}^{*}+\gamma \boldsymbol{c}^{*}$ with $\alpha$ and $\gamma$ temperature dependant and equal to 0.182 and 0.318 , respectively, at $300 \mathrm{~K}$. The superspace group is then $\mathrm{C} 2 / \mathrm{m}(\alpha 0 \gamma)$. The superspace groups have been listed at dimension $\mathrm{D}=3+1,3+2$ and $3+3$. There are 756 superspace groups for modulated structures of dimension $\mathrm{D}=3+1$. They can be found at the following internet address: http://stokes. byu.edu/iso/ssg.php.

\subsubsection{The aperiodic composites}

The aperiodic composites are formed by the intergrowth of two or more sublattices. In these crystals, aperiodicity may appear quite naturally when the lattice periodicities of the subsystems cannot adjust to each other at least in one direction. Well-known examples are the inorganic misfit layers (Fig. 2), two dimensional "sandwich" compounds where in different layers the parameters do not match [12]. The aperiodic composite can be seen as the imbrication of two incommensurately modulated sub-systems. Each subsystem plays 

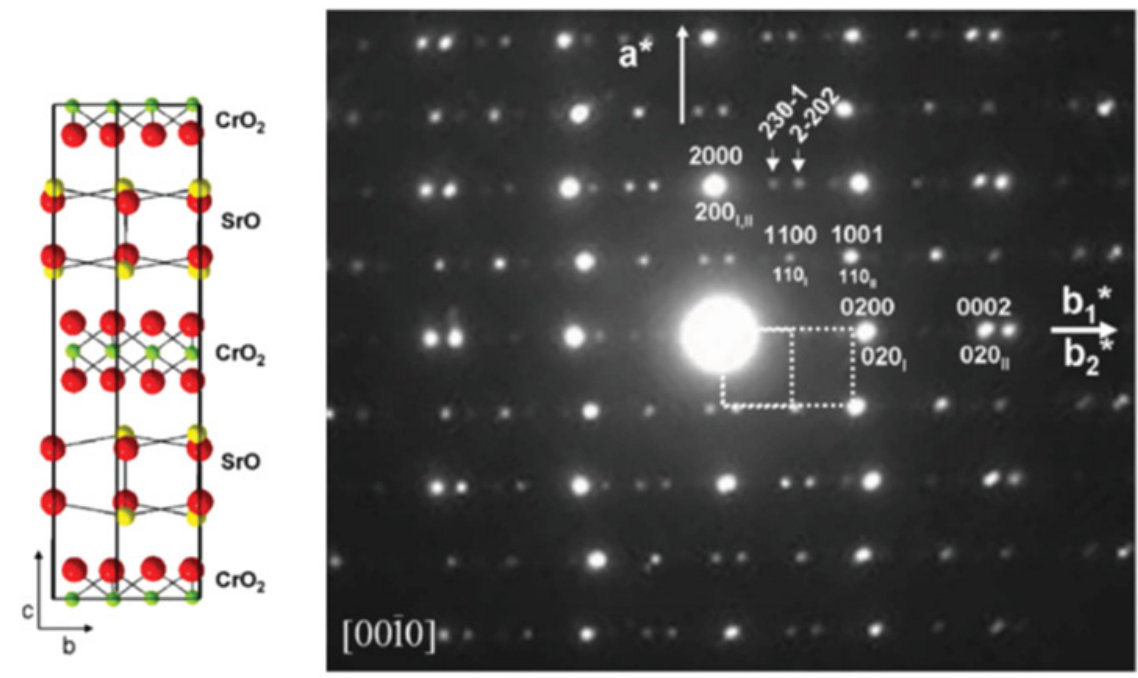

Figure 2. A two dimensional composite structure $\left[\mathrm{Sr}_{2} \mathrm{O}_{2}\right]\left[\mathrm{CrO}_{2}\right] 1.85$ with its electronic diffraction image indexed with 4 indices [19].

the role of an external potential for the other(s) sub-system(s). The major difference with an incommensurate modulated crystal is that aperiodic composites are usually aperiodic by construction: there is no high symmetry periodic phase, no mean periodic structure and no mean Brillouin zone.

In the case of a single incommensurate direction, the crystal must be described within a superspace of rank 3+1 [20]. The lattice possesses four characteristic periodicities $\mathbf{a}, \mathbf{b}$, $\mathbf{c}_{1}$ and $\mathbf{c}_{2}$, where $\mathbf{a}$ and $\mathbf{b}$ are the two vectors of the commensurate plane (perpendicular to the aperiodic direction, $\mathbf{c}_{1}$ and $\mathbf{c}_{2}$ the basis vectors of the two sublattices 1 and 2 along the incommensurate direction. Some examples of prototype materials are listed below and illustrated in the Fig. 3:

- the mercury chains in an $\mathrm{AsF}_{6}$ lattice, $\mathrm{Hg}_{3-\delta} \mathrm{AsF}_{6}$ with $\delta$ of the order of 0.20 [21].

- "self hosting" composites where the same atoms in host and guest, sublattices [22].

- the tetrathiafulvalene salts TTF-Br $r_{\mathrm{x}}$ and TTF- $\mathrm{I}_{\mathrm{x}}$ with $\mathrm{x}$ of the order of 0.79 and 0.71 respectively [23, 24].

- host/guest inclusion compounds of urea/alkane.

In the superspace, the crystal pattern is the imbrication of two atomic surfaces associated to the atoms of the two lattices. In order to describe the whole set of reciprocal vectors of the reciprocal space, one needs four reciprocal basic vectors $\mathbf{a}^{*}, \mathbf{b}^{*}, \mathbf{c}_{1} *$ and $\mathbf{c}_{2} *$. Contrary to the case of incommensurate modulated crystals, there is no more the notion of main reflection. In the present case, there are four types of Bragg peaks (Fig. 4):

- (hk00): common Bragg peaks defining the commensurate reciprocal plan (a*, $\left.\mathbf{b}^{*}\right)$.

- (hkl0): Bragg peaks reflecting the periodicity the first sublattice 1 .

- (hk0m): Bragg peaks reflecting the periodicity the second sublattice 2 .

- (hklm) with 1 and $\mathrm{m} \neq 0$ : intermodulation satellite Bragg peaks appearing at combinatory positions of $\mathbf{c}_{1} *$ and $\mathbf{c}_{2} *$ and reflecting the intermodulation between the two sublattices. 

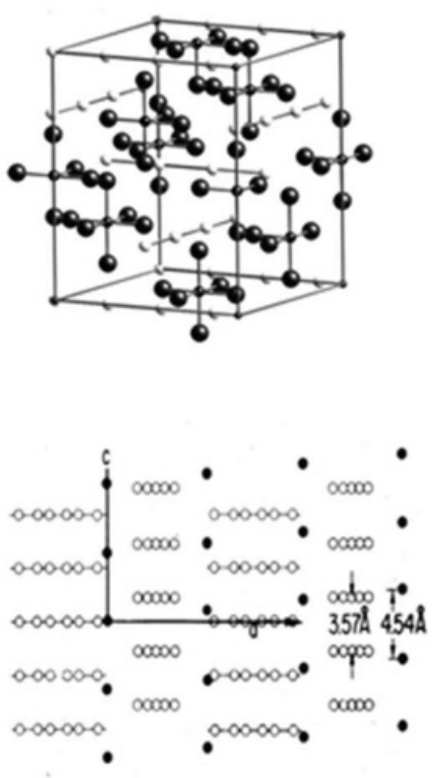
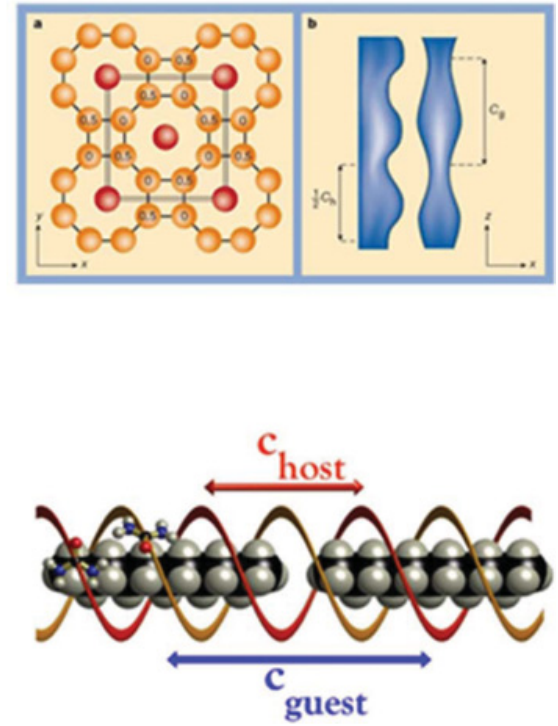

Figure 3. Examples of aperiodic composites $\mathrm{Hg}_{3-\delta} \mathrm{AsF}_{6}$, Barium self-hosted in its phase "IV", tetrathiafulvalene salt TTF- $\mathrm{Br}_{x}$ and urea/n-alkane inclusion compound.

In fact, both sublattices contribute to the intensity of the whole set of Bragg peaks. This makes the structure resolution much more complicated than in the case of incommensurate modulated crystals.

For the determination of the superspace group of an aperiodic composite, the first step is to determine the pseudo-space groups and superspace groups associated to the different subsystems considered separately [25]. The superspace group associated to the whole composite will be the largest common subgroup between the superspace groups of the different sub-systems. The complete list of superspace groups for uniaxial aperiodic composites of dimension $3+1$ has been given by van Smaalen and Harris [26].

Since aperiodic composites are aperiodic by constructions, they are very much adapted to study symmetry breakings within crystallographic superspaces. The prototype family of alkane/urea compounds has revealed very original sequences of phases in these crystals: a phase transition doubling only the internal space of the superspace by increasing the dimension of the crystallographic superspace [27, 28], the creation of monoclinic intermodulated aperiodic composites [29]. The use of combined coherent neutron scattering and high resolution synchrotron studies permits the analysis of very original pretransitional phenomena within the crystallographic superspace [30].

\subsubsection{Quasicrystals}

During a long period, crystallography considered crystals as a covering of the space resulting from the arrangement of one type of polyhedra, copied then to infinity by translation. This rule constrains the unit cell to have rotational symmetry of the order 2, 3, 4 and 6. However, more complex complex ways of covering the space have been imagined. A nice illustration is given by the Fibonacci chain where one considers an atom $\mathrm{A}$ and an atom $\mathrm{B}$. A trivial periodic tiling is obtained by simple juxtaposition of the object AB: ABABAB. A more complex process, 


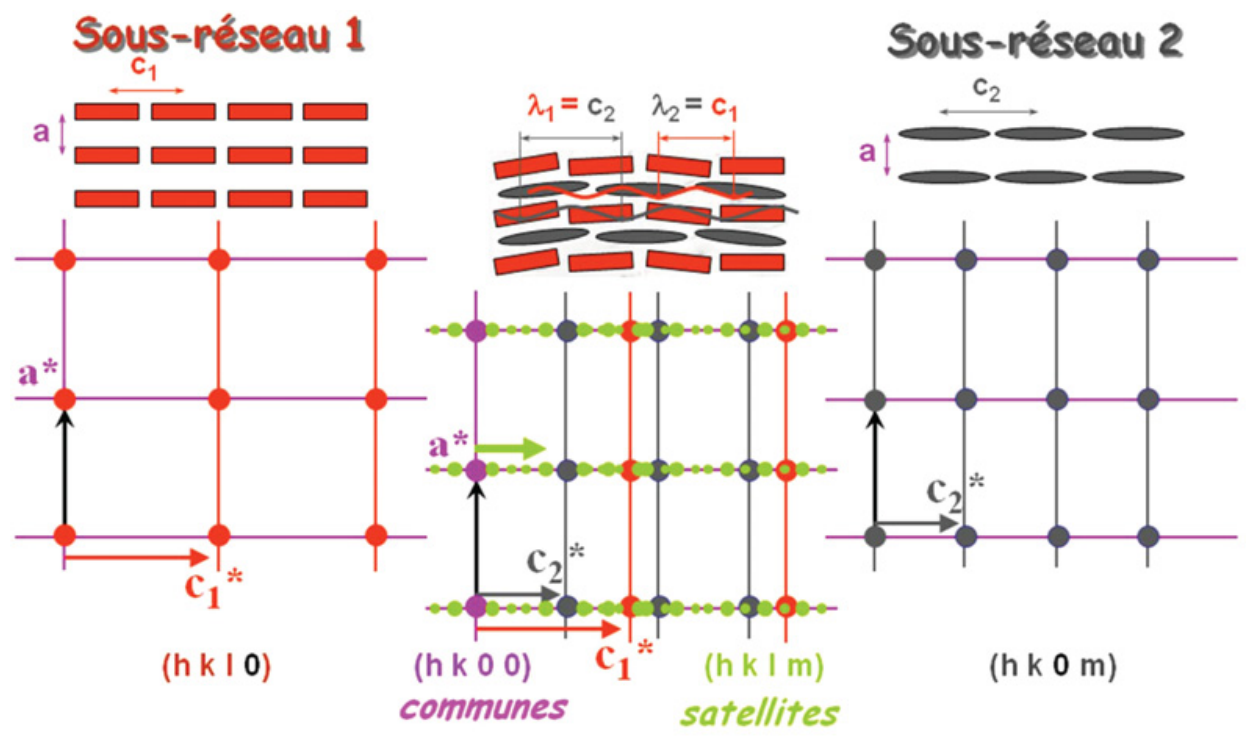

Figure 4. A composite crystal results from at least two imbricated and intermodulated sublattices 1 and 2. The diffraction image is not the simple superposition of the two independent diffraction images (right and left) but much more Bragg peaks, satellites in green, are generated by the intermodulation.

called inflation rule, where each arrangement $S(n)$ of the step $n$ is the juxtaposition of the arrangements $\mathrm{S}(\mathrm{n}-1)$ and $\mathrm{S}(\mathrm{n}-2)$, yields a long range order but without translation symmetry (Fig. 5):

\section{A, AB, ABA, ABAAB, ABAABABA, ABAABABAABAAB, etc..}

Fibonacci chain appears as a cut of a square lattice plus projection. It is easily shown that the ratio of the number of the atoms $\mathrm{A}$ and $\mathrm{B}$ is equal for an infinite chain to the golden number $\tau=2 \cos \left(\frac{2 \pi}{10}\right)$. The two dimensional equivalent is given by a tiling with two lozenges of base $\frac{2 \pi}{10}$ and $\frac{2 \pi}{15}$. This tiling created by R. Penrose in 1978 is also non-periodic. It can be shown that it is the intersection of a four dimensional periodic superspace. The three dimensional representation is closed to the one encountered in some real quasicrystals. These quasicrystals may present five-fold (pentagonal), 8 fold (octagonal), 10 fold (decagonal) or 12 fold (dodecagonal) symmetries (Fig. 6). More complexes tiling may also exist [13]. The experimental discovery of quasicrystals in 1984 was presented in the Physical Review letters with the title: "Metallic Phase with long range orientational order and no translational symmetry" by D. Schechtman, I. Blech, D. Gratias and J. W. Cahn. It was awarded by the Nobel Prize of Chemistry in 2011. We know today hundred quasicrystalline structures, mainly ternary metallic alloys often with aluminium. These quasicrystals are described within superspaces of rank up the 6 . Solving the structure of a quasicrystal is determining the shape of the discontinuous atomic hypervolumes [31, 32]. The problem is of course very complex. The study of approximant commensurate phases allows sometime to tackle the problem in a simpler way [33]. Another efficient approach is to modelise the structure of a quasicrystal by a limited number of elementary blocks or "clusters" [34, 35].

The existence of diffuse scattering in the quasicrystal diffraction image is quite usual. Of course, there is the same type of static (substitution, occupation and displacement) and dynamic (phonons and atomic diffusion) disorder than in usual periodic crystals. Other 
a)

A B

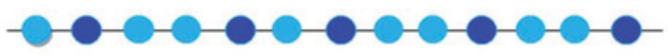

c)

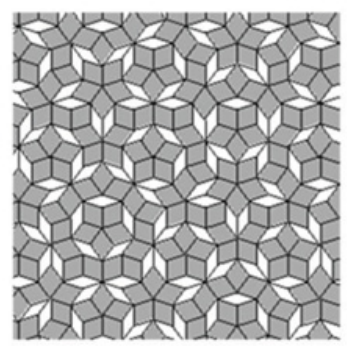

b)

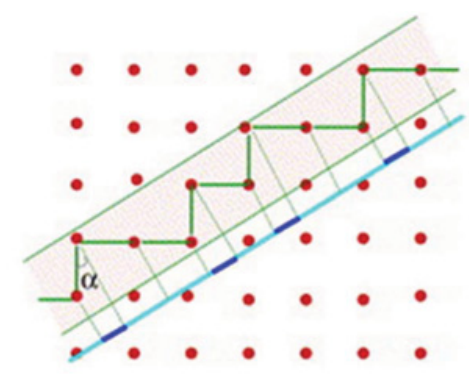

d)

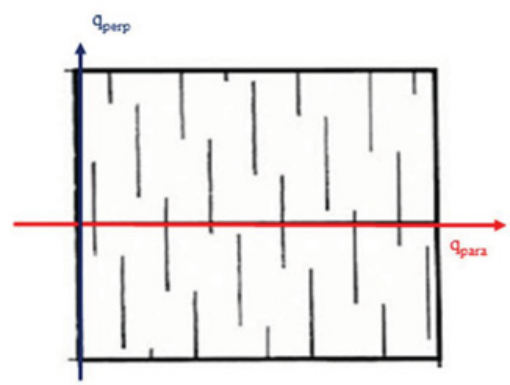

Figure 5. a) Fibonacci chain of atoms A and B;. b) A 1D aperiodic Fibonacci chain is obtained by a cut of a periodic two dimensional square lattice; c) A Penrose tiling, the two dimensional analog of the Fibonacci chain; d) the atomic function corresponding to a quasicystal is given by finite segments along the internal space of the crystallographic superspace.

kinds of disorder are specific to quasicrystals and are related to the difficulty in defining a simple mean structure. Let us note that until now, there is a discussion on the origin of the stabilization of the quasicrystals either by the energy or by the entropy. In that last case, the quasicrystals would transform in a periodic approximant on approaching zero Kelvin, whereas in the first case the quasicrystalline state would be the fundamental state. The very slow kinetic of the metallic structures at low temperature does not allow to answer this question. This complexity appears in all studies of phase transitions in these materials, and consequently few examples illustrate the broken symmetry in quasicrystals.

\subsection{Dynamics in aperiodic crystals}

\subsubsection{Incommensurate modulated crystals}

The incommensurate modulated crystals phases are the simplest aperiodic crystals and they were extensively studied in the 80's [15]. The simplicity in these materials comes from the fact that they have a high symmetry periodic phase. The Brillouin zone of these periodic crystals will play the role of the mean Brillouin zone in the incommensurate phase. In that phase, a huge majority of the phonon branches remain unchanged compared to the periodic crystal. The Fig. 7 summarizes the structural characteristics of an incommensurate crystal which possesses a high symmetry periodic phase and a lock-in low temperature commensurate phase.

The basis from the intermediate incommensurate phase of rank four, characterized by an unique critical modulation vector $\mathbf{q}_{\mathrm{s}}$, is described by the vectors $\mathbf{a}^{*}, \mathbf{b}^{*}, \mathbf{c}^{*}$ and $\mathbf{q}_{\mathrm{s}}$. The static modulation appearing in the incommensurate phase, couples the phonon branches degenerated in $\pm \mathbf{q}_{s}$, giving rise to two new modes a symmetric one, the amplitudon, and an asymmetric one, the phason [37]. Referring to displacive phase transition governed 


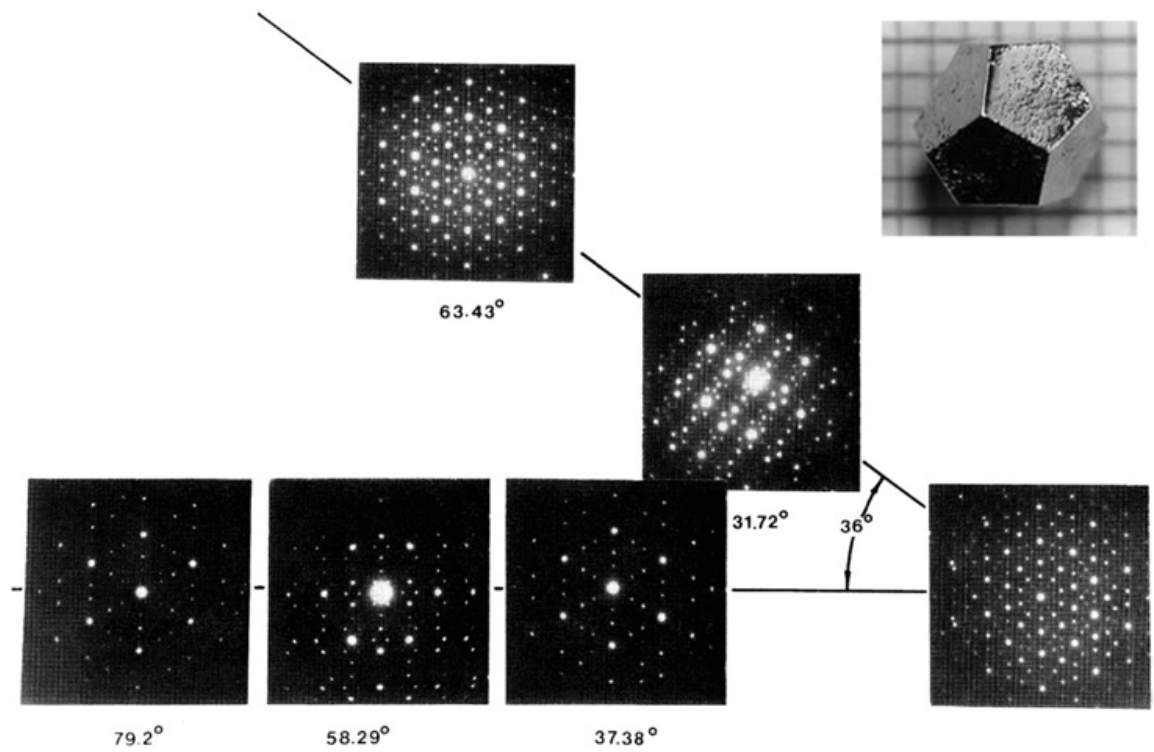

Figure 6. Electronic diffraction image of an icosahedral quasicrystal [36]. In inset, an icosahedral quasicrystal of $\mathrm{Zn}-\mathrm{Mg}-\mathrm{Ho}$.

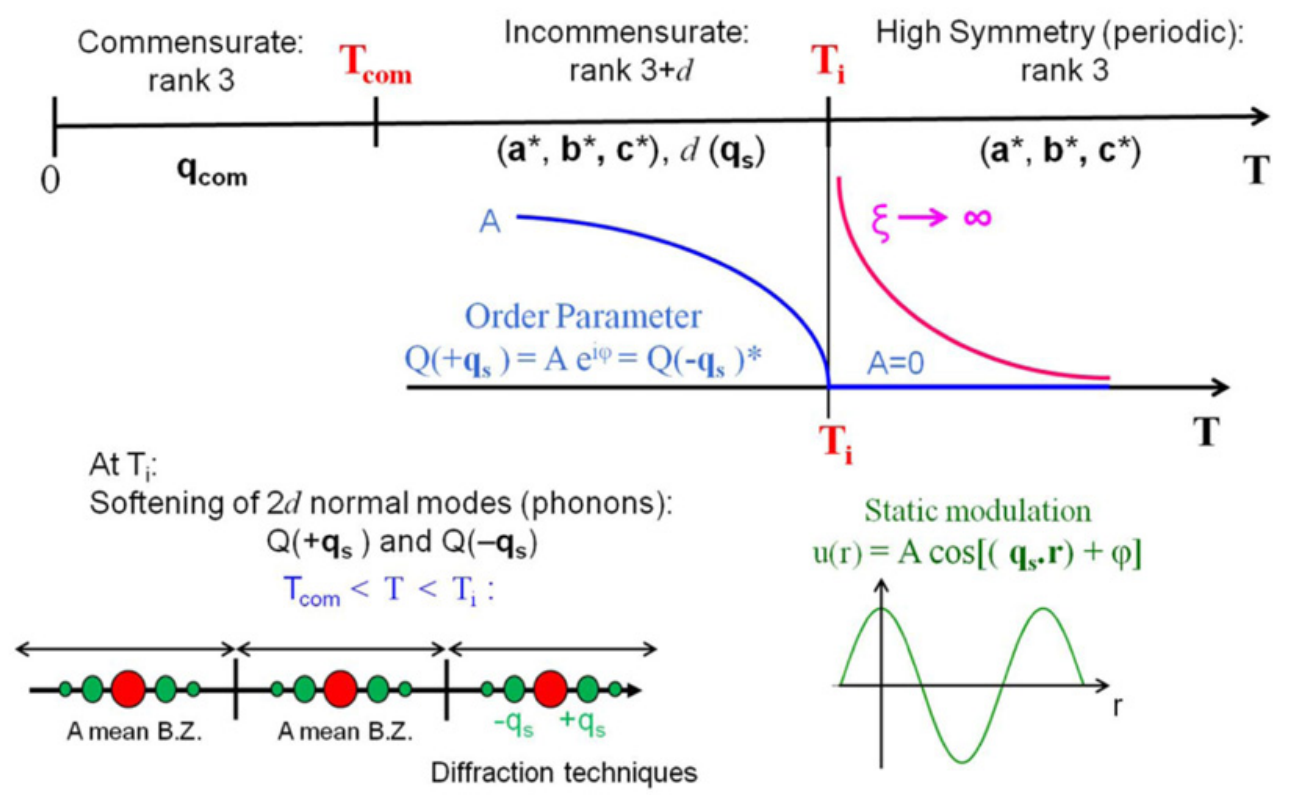

Figure 7. Schematic representation of the sequences of phase in a modulated incommensurate crystal which possess a periodic high symmetry phase. At the transition temperature $\mathrm{T}_{\mathrm{i}}$, the correlation lengths $\xi$ diverge and below $\mathrm{T}_{\mathrm{i}}$ one defines an order parameter as a complex quantity $\mathrm{Q}\left(\mathbf{q}_{\mathrm{s}}\right)=\mathrm{Ae} \mathrm{e}^{i \varphi}=\mathrm{Q}\left(-\mathbf{q}_{\mathrm{s}}\right)^{*}$. The incommensurate phase is characterized by the static modulation $\mathrm{u}(\mathrm{r})=\mathrm{A} \cos \left[\left(\mathbf{q}_{\mathrm{s}} \cdot \mathrm{r}\right)+\varphi\right]$ where $\mathrm{A}$ is the amplitude and $\varphi$ the phase of the modulation. The existence of the static modulation induces the existence of satellite Bragg peaks at positions $\pm \mathrm{nq}_{\mathrm{s}}$ (in green) around the high symmetry Bragg peaks (in red). Often, at lower temperature, there is a lock-in phase transition towards a commensurate phase at $\mathrm{T}_{\text {com. }}$. 


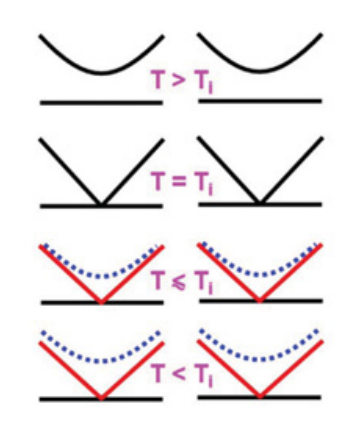

The static modulation couples degenerated modes at $2 \mathbf{q}_{\mathbf{s}}$

$$
\begin{gathered}
\text { Dispersion around } \mathbf{q}_{\mathrm{s}} \\
\text { Amplitudon: } \\
\begin{array}{c}
\omega_{\mathrm{A}}{ }^{2}(\mathbf{q})=\omega_{\mathrm{A}}{ }^{2}\left(\mathbf{q}_{\mathrm{s}}\right)+\mathrm{D} \mathrm{q}^{2} \\
\text { Phason: } \\
\omega_{\mathrm{Ph}}(\mathbf{q})=\mathrm{V}_{\mathrm{Ph}} \cdot \mathbf{q}
\end{array}
\end{gathered}
$$
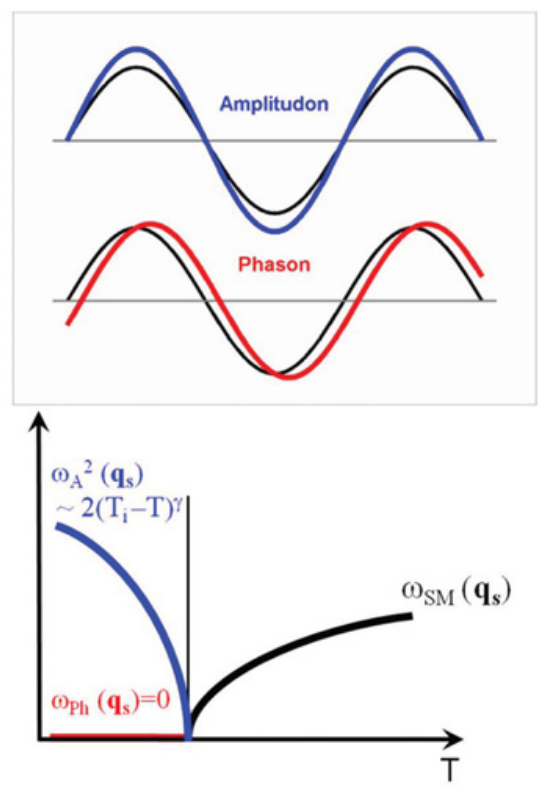

Figure 8. Collective dynamics around a displacive phase transition to an incommensurate modulated phase. Below $\mathrm{T}_{\mathrm{i}}$, the static modulation couples degenerated modes separated by $2 \mathbf{q}_{\mathrm{s}}$ creating two excitations the amplitudon (usual soft mode) in blue and the phason (zero energy mode at $\mathbf{q}_{\mathrm{s}}$ ) in red.

by a soft phonon branch in the high symmetry phase (Fig. 8), the amplitudon within the incommensurate phase is the usual low symmetry soft mode. In the same way, it presents the following dependencies in $\mathbf{q}_{\mathrm{s}}$ and around $\mathbf{q}_{\mathrm{s}}: \omega_{A}^{2}\left(\boldsymbol{q}_{s}\right) \propto 2\left(T_{i}-T\right)^{\gamma}$ and $\omega_{A}^{2}(q)=$ $\omega_{A}^{2}\left(\mathbf{q}_{\mathbf{s}}\right)+D q^{2}$. The associated phason mode presents the linear dispersion, going from zero energy at $\mathbf{q}_{\mathrm{s}}$ in the whole incommensurate phase $\omega_{P h}^{2}\left(\mathbf{q}_{\mathrm{s}}\right)=0$ and $\omega_{P h}(\boldsymbol{q})=V_{P h} . \boldsymbol{q}$. The phason mode corresponds to a fluctuation along the internal space of the superspace. It is like a supplementary Goldstone-like mode with a liner dispersion as an acoustic phonon (in the case of a superspace of rank $\mathrm{D}=3+\mathrm{d}$, $\mathrm{d}$ such branches are predicted). It should be mentioned that even if these modes present a linear dispersion in zone center within the whole incommensurate phase, they have a finite damping at $\mathbf{q}_{\mathrm{s}}$ contrary to real Goldstone modes (acoustic mode at Bragg position). The reason is that these phason modes correspond to a relative displacement of the atoms (which means dissipation) which is not the case for acoustic phonons. Experimentally, this last property explains why phason modes are so efficient to the spin-lattice relaxation rate in NMR spectroscopy and indeed many incommensurate phases where discovered by this technique. However, coherent neutron scattering was the unique way to measure the phason spectral function getting by the way frequency dispersion and damping of this excitation [15, 38-41].

\subsubsection{Dynamics in aperiodic composites}

The collective dynamics in aperiodic composite is very complex because of two main reasons. The first one is the absence of a high symmetry periodic phase, which makes impossible the calculation for a finite number of atoms as in incommensurate modulated crystals. The second reason is that it is impossible to define even a mean Brillouin zone, and so the real Brillouin zone is effectively of zero volume. Such a result is particularly well illustrated by 
a)
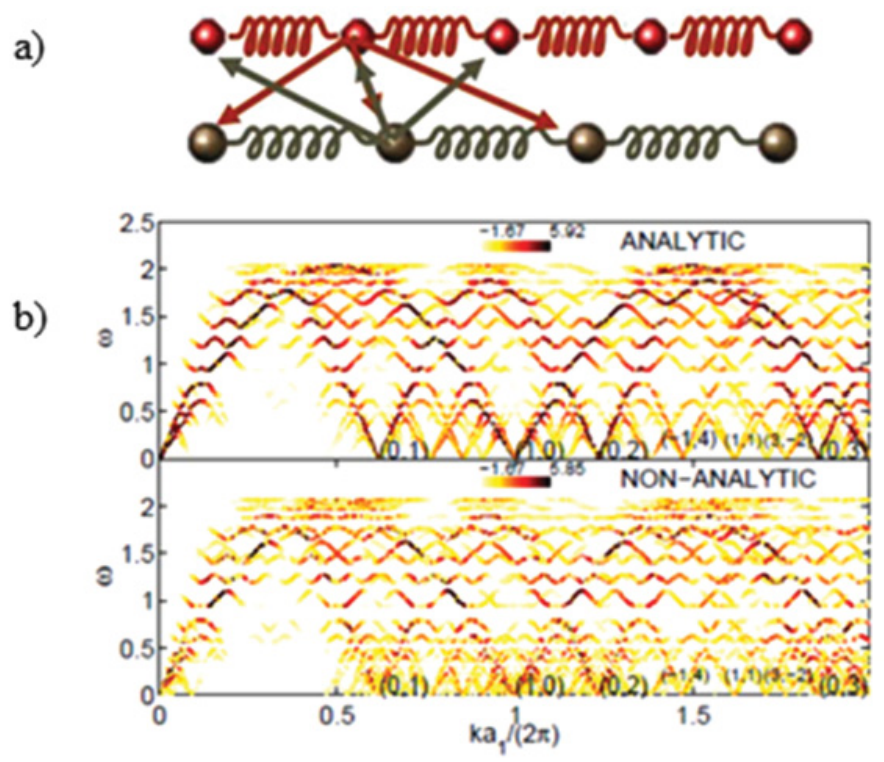

Figure 9. Collective dynamics in an aperiodic composite crystal modelled by coupled deformable chains (up). The phonon branches are shown in the case an analytic and of a non-analytic atomic function. The colors show the dynamical structure factor on a logarithmic scale [42].

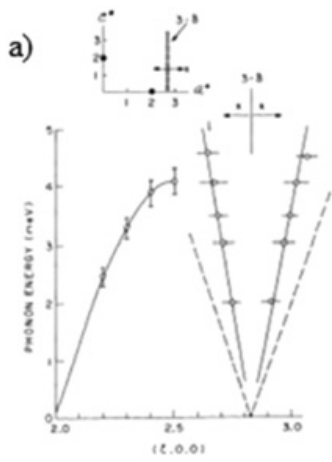

b)

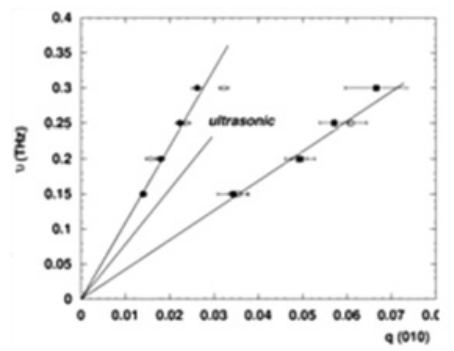

c)

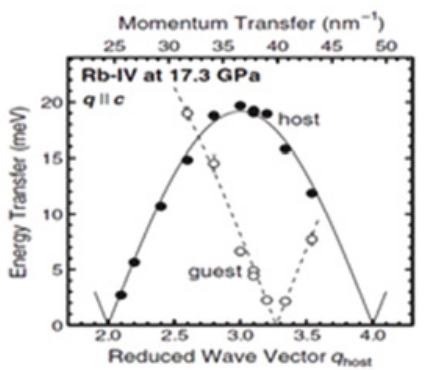

Figure 10. Longitudinal acoustic phonon branches measured along the aperiodic direction, a) in mercury chain $\mathrm{Hg}_{3-\delta} \mathrm{AsF}_{6}$, b) in $\mathrm{Bi}_{2} \mathrm{Sr}_{2} \mathrm{CuO}_{6+x}$ and c) in the "self-hosting" crystal of rubidium in its phase "IV".

the calculation of deformable coupled aperiodic chains by T. Janssen et al. [42], as shown in the Fig. 9.

The analytical resolution of the equation of motion leads the dispersion branches shown in the figure. Two properties come out: the emergence of phonon branches out of the four type of Bragg peaks defined previously for a one dimensional aperiodic composite (hk00), (hkl0), (hk0m) and (hklm). Aperiodicity can be seen as resulting from an infinite folding of the dispersion branches with multiple energy gaps opening in these branches. The curves obtained experimentally appear much more simples than the ones predicted theoretically (Fig. 10). The reason is that the inelastic structure factors are in most case too weak and only allows the observation of the phonon branches emerging from structure common, and each 
a)

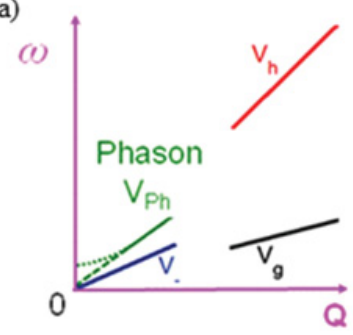

c)

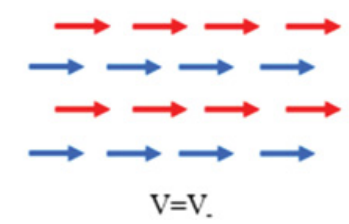

b)

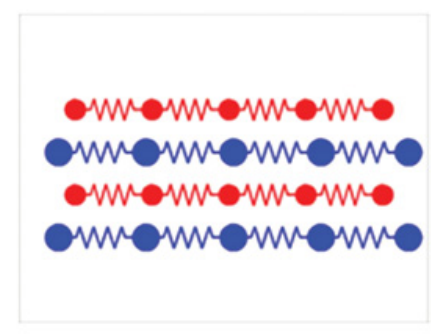

d)

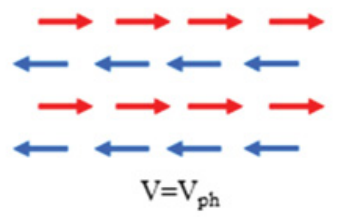

Figure 11. (a) Dispersion branches of two sublattices $\left(\mathrm{V}_{\mathrm{h}}\right.$ and $\left.\mathrm{V}_{\mathrm{g}}\right)$ coupled close to $\mathrm{Q}=0$ to give acoustic phonon branch of slope $V_{-}$and a phason branch of slope $V_{p h}$, without gap in energy if the atomic function is analytical (b), (c) et (d) Schematic representation of the components of the motions in phase for the acoustic phonon (a unique speed of sound in the crystal), in antiphase for the phason ("sliding mode").

sublattice Bragg peaks. In some way, the obtained image experimentally corresponds solely to the signature of the collective dynamics of the two quasi-independent sublattices ignoring the influence of their intermodulation. So, each Bragg peaks yields the elastics constant of its on sublattice, force constant defining the slope of the longitudinal acoustic phonon branch in the incommensurate direction. Two rather different slopes were observed in $\mathrm{Bi}_{2} \mathrm{Sr}_{2} \mathrm{CuO}$ [43]. In the "self-hosting" composite, where the same atom is implicated both in the host and in the guest matrix, it is quite naturally the same slope which is measured around (hk10) and (hk0m) Bragg peaks [44]. In the high symmetry phase of $\mathrm{Hg}_{3-\delta} \mathrm{AsF}_{6}$, the mercury presents a one dimensional liquid-like character to which are associated scattering plane perpendicular to the incommensurate direction (in this case, actually two orthogonal directions). The coherent neutron scattering measurements has permitted the observation of two branches with totally different slopes associated respectively to the host $\mathrm{AsF}_{6}$ matrix and the guest quasi-liquid mercury [45].

Axe and Bak have shown that at the origin of the reciprocal space of an aperiodic composite the two branches of the host and guest couple together to generate two new excitations $[42,46,47]$. One branch is the usual acoustic branch corresponding to an in phase displacement of the atoms of both subsystems. The slope of this branch defines the unique speed of sound in these aperiodic crystals. The other branch is associated to fluctuations along the internal space of the crystallographic superspace and is a phase fluctuation mode (Fig. 11). This antisymmetric mode keeps the center of mass of the two sublattices when vibrating in antiphase. In aperiodic composites, this phason mode is usually called "sliding mode". No direct observation of such a sliding mode has been done yet.

A recent study [48] reported the coupling of the longitudinal acoustic mode of the host subsystem with such a sliding mode (Fig. 12). Measurement was performed by neutron scattering. It was done on an organic host-guest compound $n$-nonadecane/urea. Phonon branches were measured along the incommensurate direction close to a strong host (urea) 

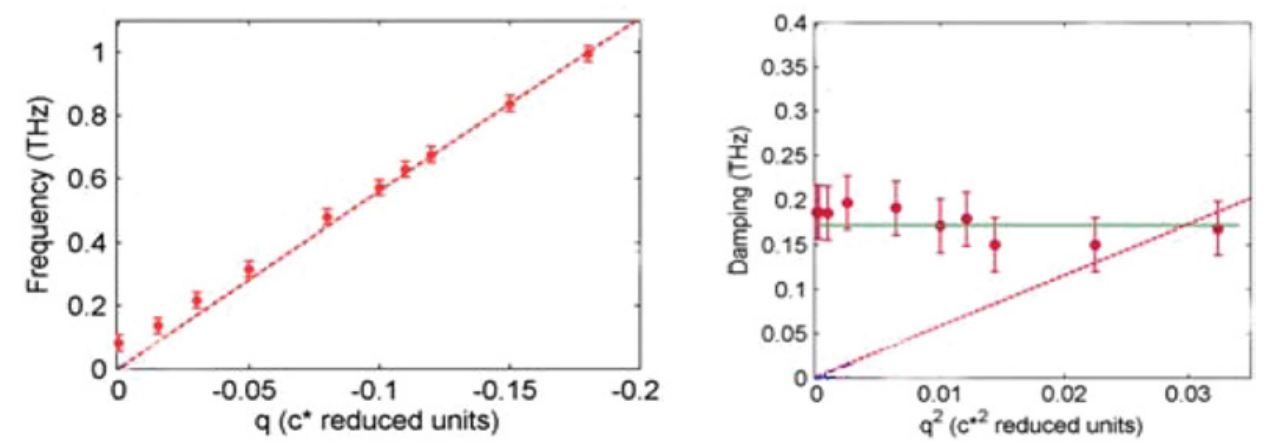

Figure 12. Neutron study of the coupling of the longitudinal acoustic mode of the host subsystem with a sliding mode in $n$-nonadecane/urea. It reveals an energy gap on the L.A. phonon branch together with a non-vanishing damping of this mode on approaching the Bragg position [48].

Bragg peaks. It reveals two new features: an energy gap on the L.A. phonon branch together with a non-vanishing damping of this mode on approaching the Bragg position. This measurement is an indirect evidence of the influence of the phason mode on usual acoustic phonons in an aperiodic crystal. Much more studies have to be done in aperiodic composites before getting a full understanding of the collective dynamics in such crystals without Brillouin zone.

\subsubsection{Dynamics in quasicrystals}

The determination of the collective dynamics in the quasicrystals is certainly one of the most complex problems in crystals. So, the calculation of the phonon branches is more easily done by considering commensurate approximants. The Fibonacci chain appears as a simple one dimensional representation of a quasicrystal. Its diffraction image is made of Bragg peaks and the phonon branches appear like emerging from the most intense Bragg peaks, which somehow play the role of zone center $\Gamma$ [49]. The simplest periodic approximation is the simple repetition here of long L and short S segment: LSLSLS. The dispersion branches corresponding to this arrangement is presented in the Fig. 13. Such an image can be generalized to quasicrystals where Bragg peaks of 5 and 10 fold symmetry could play the role of zone center where acoustic phonon branches will emerge. Reciprocal image of a quasicrystal requires the use of 4 to 6 indices depending on the dimension of its crystallographic superspace. Again, physical space and internal space (often called perpendicular space) are defined. Around the nodes of the physical reciprocal space is measured the diffuse scattering called "Thermal Diffuse Scattering" (TDS) associated to phonons. Around the nodes of the perpendicular space is measured the diffuse scattering called "Phason Diffuse Scattering" (PDS) associated to phason modes. The nature of quasicrystals, aperiodic materials described in crystallographic superspaces by discontinuous atomic surfaces, generates other types of dynamics and disorder in addition to the hydrodynamics modes relative to TDS and PDS. So, diffusive modes associated to atomic jumps ("dynamic phason flip") where evidenced by local probes such as N.M.R. and incoherent neutron scattering [50, 51]. An extensive study of phonon and phason modes in quasicrystals where realized by M. de Boissieu (Fig. 14) and other teams. Such experimental studies require large prototypical quasicrystals. These results are discussed in several books and articles. 

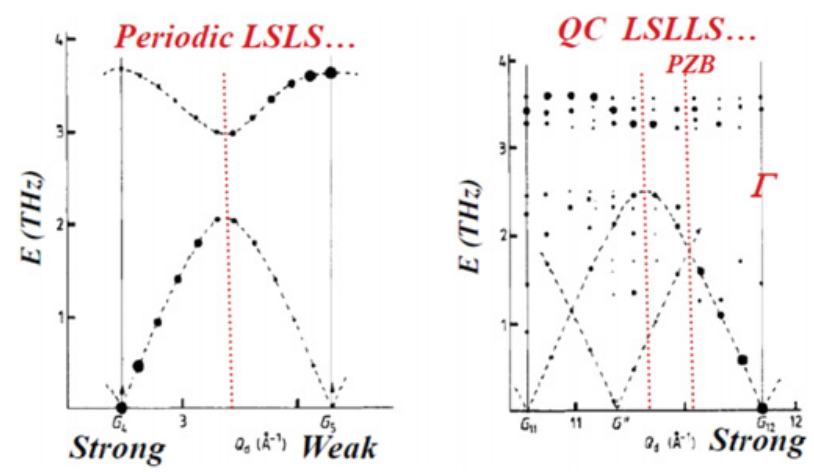

Figure 13. Dispersion branches for a periodic crystal (left) and for a quasicrystal described by a Fibonacci chain (right). In that last case, the strong Bragg peaks play the role of a center of zone $\Gamma$ of a pseudo Brillouin Zone (PZB) [49].
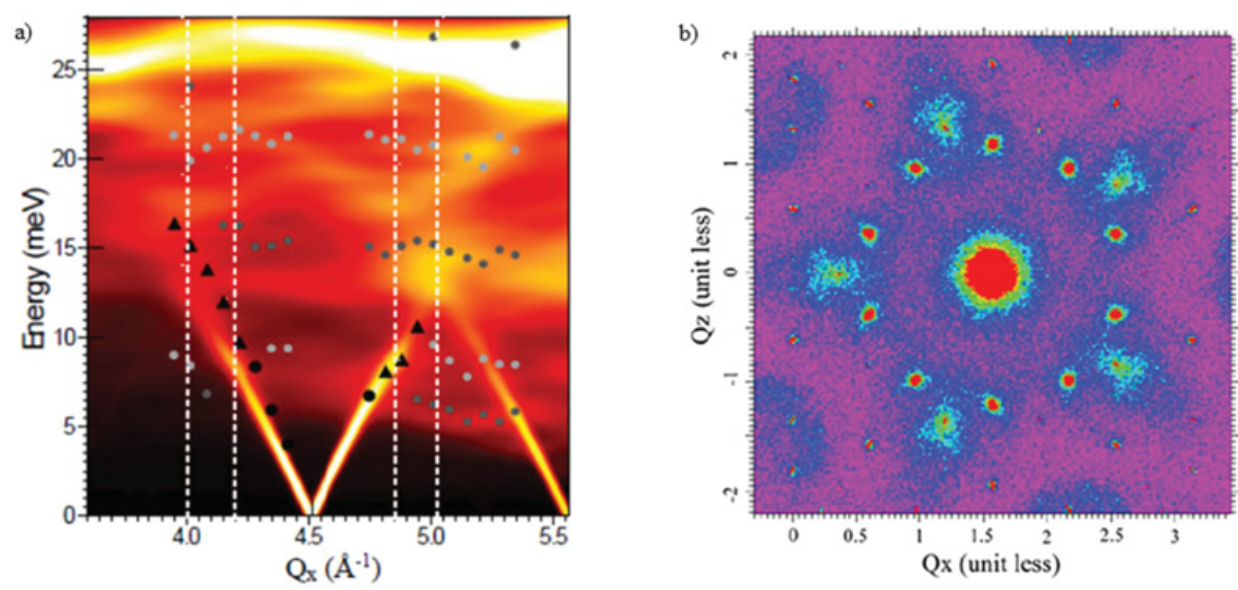

Figure 14. a) Longitudinal modes measured by $\mathrm{X}$-ray inelastic scattering in the quasicrystal $\mathrm{ZnMgSc}$ [52] b) Diffuse scattering in the plane perpendicular to the 5 fold symmetry of the i-AlPdMn [53].

\section{Conclusion}

Neutron scattering has played a major role in the understanding of the order and the dynamics in solid state matter since the middle of the twentieth century. Since the discovery of aperiodic crystal neutron diffraction coupled to X-ray and synchrotron diffraction has allowed the generalization of the standard crystallography towards superspace crystallography. It appears then that a periodic crystal is a particular case of a more general definition of the crystal. It is obtained when the number independent vectors needed to described the periodic cell is equal to the dimension of the physical space (we then say that the rank of the space is equal to the dimension of the space). In aperiodic crystals the rank is larger than the physical space. The crystallographic superspace then decomposes in two spaces the physical space and the internal space (sometime called perpendicular space). Within this superspace, translation symmetry is recovered, defining a supercell and complex atomic functions within this supercell. The structural solution results from a physical cut of this high dimensional space. Many more space groups can be generated by increasing the rank of the superspace. 
This offers to the aperiodic crystal an increase of the possible structural solution. Neutron diffraction appears there as an essential technique to study the phase transition within crystallographic superspaces. These phase transitions are associated to critical phenomena and critical dynamics. Coherent neutron scattering is the most adapted technique to measure the collective excitation in these materials which do not possess any Brillouin zone.

\section{References}

[1] L. Landau, E. Lifchitz, Physique Statistique (Editions MIR, Moscou, 1964)

[2] R. Cowley, Adv. Phys. 29(1), 1-110 (1980)

[3] A. Bruce, Adv. Phys. 29, 111 (1980)

[4] A. Bruce, R. Cowley, Adv. Phys. 29, 219-321 (1980)

[5] A. Levanyuk, Sov. Phys. Solid State 5, 1294-1298 (1964)

[6] P.M. de Wolff, Acta Cryst. A 30, 777 (1974)

[7] P.M. de Wolf, Acta Cryst. A 33, 493-497 (1977)

[8] P.M. de Wolff, T. Janssen, A. Janner, Acta Cryst. A 37, 625-636 (1981)

[9] T. Janssen, Acta Cryst. A 68, 667-674 (2012)

[10] T. Janssen, G. Chapuis, M. de Boissieu, Aperiodic Crystals, From modulated phases to quasicrystals (Oxford science publication, International Union of crystallography, 2007)

[11] S. van Smaalen, Z. für Kristallogr. 219, 681-691 (2004)

[12] S. van Smaalen, Incommensurate Crystallography (Oxford Science Publication, International Union of Crystallography, 2009)

[13] D. Gratias, R. Mosseri, J. Prost, J. Toner, M. Duneau, Du cristal à l'amorphe (Éditions de Physique, Les Ulis, 1988)

[14] A. Herpin, P. Mériel et J. Villain, Compt. Rend. Acad. Sciences 249, 1334 (1959)

[15] R. Blinc, A. Levanyuk, Incommensurate phases in dielectrics (North Holland, 1986)

[16] P. Monceau, Adv. Phys. 61, 325-581 (2012)

[17] T. Janssen, J. Phys.: Condens. Matter 14, 12411 (2002)

[18] T. Janssen, O. Radulescu, A. Rubtsov, Eur. Phys. J. B 29, 136 (2002)

[19] E. Castillo-Martinez, A. Schönleber, S. van Smaalen, A.M. Arevalo-Lopez, M.A. Alario-Franco, J. Solid State Chem. 181, 1840-1847 (2008)

[20] P. Coppens, Acta Cryst. B 51, 402-410 (1995)

[21] J.P. Pouget, G. Shirane, J.M. Hastings, A.J. Heeger, N.D. Miro, A.G. MacDiarmid, Phys. Rev. B 18, 3645-3656 (1978)

[22] R.J. Nelmes, D.R. Allan, M.I. McMahon, S.A. Belmonte, Phys. Rev. Lett, 83, 4081-4084 (1999)

[23] J.B. Torrance, B.A. Scott, B. Welber, F.B. Kaufman, P.E. Seiden, Phys. Rev. B 19, 730 (1979)

[24] C.K. Johnson, C.R. Watson, J. Chem. Phys. 64, 2271 (1976)

[25] S. van Smaalen, Phys. Rev. B 43, 11330 (1991)

[26] S. van Smaalen, K.D.M. Harris, Superspace group description of the symmetries of incommensurate urea inclusion compounds, Proceedings: mathematical, physical and engineering sciences, p. 677-700 (1996)

[27] B. Toudic, P. Garcia, C. Odin P. Rabiller, C. Ecolivet, E. Collet, P. Bourges, G.J. McIntyre, M.D. Hollingsworth, T. Breczewski, Science 319, 69 (2008)

[28] B. Toudic, P. Rabiller, L. Bourgeois, M. Huard, C. Ecolivet, G.J. McIntyre, P. Bourges, T. Breczewski, T. Janssen, Temperature-pressure phase diagram of an aperiodic host guest compound, Europhys. Lett. 93, 16003 (2011) 
[29] C. Mariette, M. Huard, P. Rabiller, S.M. Nichols, C. Ecolivet, T. Janssen, K.E. Alquist, M.D. Hollingsworth, B. Toudic, J. Chem. Phys. 136, 104507 (2012)

[30] C. Mariette, L. Guérin, P. Rabiller, C. Ecolivet, P. García-Orduña, P. Bourges, A. Bosak, D. de Sanctis, M.D. Hollingsworth, T. Janssen, B. Toudic, Phys. Rev. B 87, $104101(2013)$

[31] C. Janot, J-M. Dubois, M. de Boissieu, Am. J. Phys. 57, 972 (1989)

[32] D. Gratias, M. Quinquandon, Philosoph. Mag. 8, 1887-1903 (2008)

[33] A. Yamamoto, H. Takakura, Phys. Rev. B 68, 132201 (2003)

[34] D. Gratias, F. Puyraimond, M. Quinquandon, A. Katz, Phys. Rev. B 63, 024202 (2000)

[35] M. Quinquandon, D. Gratias, Phys. Rev. B 74, 214205 (2006)

[36] D. Schechtman, I. Blech, D. Gratias, J. W. Cahn, Phys. Rev. Lett. 53, 1951-1953 (1984)

[37] J.D. Axe, Incommensurate Structural Phase Transformations, Proc. of Gatlinburg Conf. on Neutron Scatt., edited by Moon R., p. 353 (1976)

[38] H. Cailleau, Solid State Comm. 33, 407-411 (1980)

[39] L. Bernard, R. Currat, P. Delamoye, C.M.E. Zeyen, S. Hubert, R. de Kouchkovksy, J. Phys. C: Solid State Phys. 16, 433 (1983)

[40] P. Launois, F. Moussa, M.H. Lemée-Cailleau, H. Cailleau, Phys. Rev. B 40, 5042-5055 (1989)

[41] J. Ollivier, J. Etrillard, B. Toudic, C. Ecolivet, P. Bourges, A.P. Levanyuk, Phys. Rev. Lett. 81, 3667 (1998)

[42] O. Radulescu, T. Janssen, J. Etrillard, Eur. Phys. J. B 29, 385-398 (2002)

[43] J. Etrillard, L. Bourgeois, P. Bourges, B. Liang, C.T. Lin, B. Keimer, Europhys. Lett. 66, 246 (2004)

[44] I. Loa, L.F. Lundegaard, M.I. Mc Mahon, S.R. Evans, A. Bossak, M. Krisch, Phys. Rev. Lett. 99, 035501 (2007)

[45] J.M. Hastings, J.P. Pouget, G. Shirane, A.J. Heeger, N.D. Miro, A.G. MacDiarmid, Phys. Rev. Lett. 39, 1484-1487 (1977)

[46] J.D. Axe, P. Bak, Phys. Rev. B 26, 4963 (1982)

[47] R. Currat, E. Kats, I. Luk'yanchuk, Phys. J. B 26, 339 (2002)

[48] B. Toudic, R. Lefort, C. Ecolivet, L. Guérin, R. Currat, P. Bourges, T. Breczewski, Phys. Rev. Lett. 107, 205502 (2011)

[49] G. Poussigue, C. Benoit, M. de Boissieu, R. Currat, J. Phys. Condens. Matter 6, 659 (1994)

[50] S. Lyonnard, Etude des sauts atomiques dans les quasicristaux, Thèse de doctorat de l'Université de Paris 11 (1997)

[51] G. Coddens, W. Steurer, Phys. Rev. B 60, 270 (1999)

[52] M. de Boissieu, S. Francoual, M. Mihalkovic, K. Shibata, A.Q.R. Baron, Y. Sidis, T. Ishimasa, D. Wu, T. Lograsso, L.-P. Regnault, F. Gähler, S. Tsutsui, B. Hennion, P. Bastie, T.J. Sato, H. Takakura, R. Currat, A.-P. Tsai, Nature Mat. 6, 977 (2007)

[53] M. de Boissieu, S. Francoual, Y. Kaneko, T. Ishimase, Phys. Rev. Lett. 95, 105503 (2005) 